\title{
The Kulinkovich Reaction
}

\section{Key words}

cyclopropanes

Grignard reagent

titanium

\section{Synfact $\dot{\bar{d}}$}

Examples:

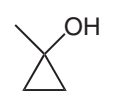

$76 \%$ yield

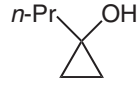

$91 \%$ yield

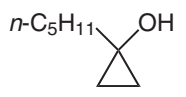

$94 \%$ yield

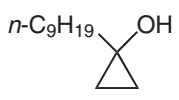

$94 \%$ yield

Reaction mechanism:
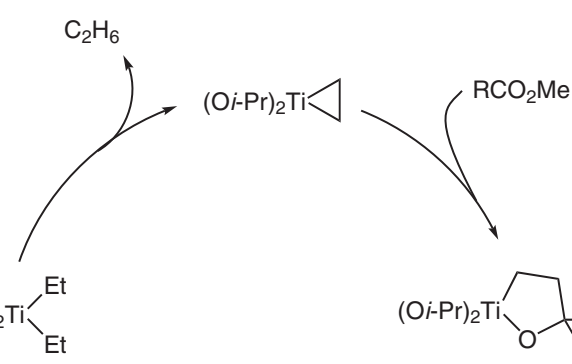

$\mathrm{Ti}(\mathrm{O} i-\mathrm{Pr})$

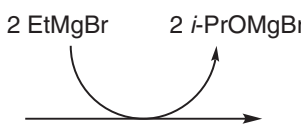
$(\mathrm{O} i-\mathrm{Pr})_{2} \mathrm{Ti}_{\mathrm{Et}}{ }^{\mathrm{Et}}$
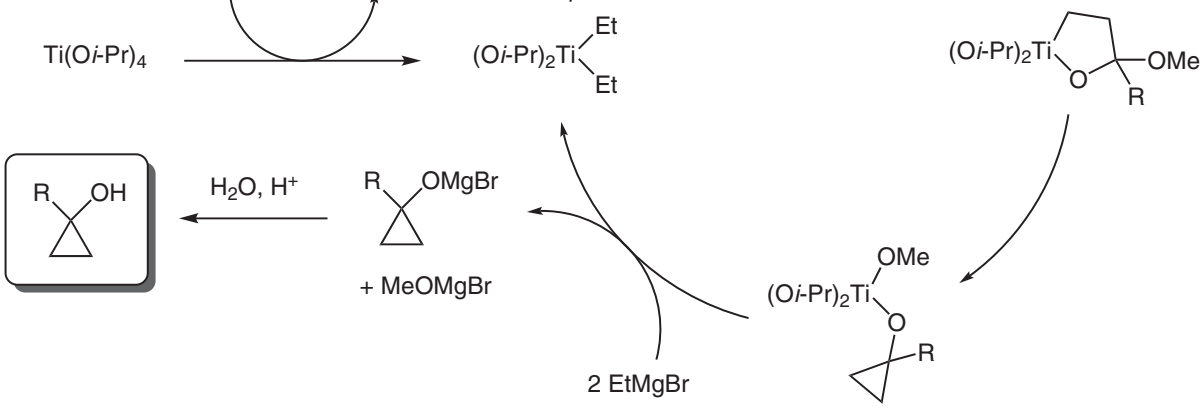

Significance: In 1991, Kulinkovich and co-workers reported the titanium-catalyzed reaction of simple esters with ethylmagnesium bromide, leading to substituted cyclopropanols in excellent yields.
Comment: Interestingly, in the presence of the titanium catalyst, ethylmagnesium bromide is transformed into an ethylene dianion, which is reactive towards alkylcarboxylic esters. 